\title{
Erratum: Causality and Loop-Tree Duality at Higher Loops [Phys. Rev. Lett. 122, 111603 (2019)]
}

\author{
Robert Runkel, Zoltán Szőr, Juan Pablo Vesga, and Stefan Weinzierl
}

(Received 26 June 2019; published 31 July 2019)

DOI: 10.1103/PhysRevLett.123.059902

In this Letter, the formula in Eq. (21) is not correct. This has been pointed out in Ref. [1]. We erroneously assumed that after averaging over all possible ways to close the contour, the representation in terms of the $|\mathcal{E}|$ local residues is unique, such that the coefficient of each residue can be computed in a suitable chosen coordinate system. This is not the case. The representation in terms of local residues depends on the initially chosen coordinate system. Of course, different representations are equivalent due to linear relations among the local residues.

The correct procedure to compute the representation in terms of local residues follows [1]: We specify a set of integration variables by $\tilde{\sigma} \in \mathcal{C}_{\Gamma}$ and an order in which the integrations are performed by $\tilde{\pi} \in S_{l}$. We assume that the integration over $k_{\tilde{\sigma}_{\tilde{\pi}_{1}}}$ is performed first, followed by the integration over $k_{\tilde{\sigma}_{\tilde{n}}}$, etc. In order to keep the indexing to a minimum, we introduce the ordered set $\tilde{k}=\left(\tilde{k}_{1}, \ldots, \tilde{k}_{l}\right)=\left(k_{\tilde{\sigma}_{\pi_{1}}}, \ldots, k_{\tilde{\sigma}_{\tilde{\pi}_{l}}}\right)$. Further, let $\tilde{\alpha}=\left(\Gamma_{1}, \ldots, \Gamma_{l}\right)$ be the ordered set of winding numbers. For a cut specified by $\sigma \in \mathcal{C}_{\Gamma}$, we denote by $\pi \in S_{l}$ the order in which the cuts are taken; e.g., the cut of the edge $e_{\sigma_{\pi_{1}}}$ is taken in the first integration, followed by the cut of the edge $e_{\sigma_{\pi_{2}}}$, etc. Again, in order to keep the indexing to a minimum, we introduce the ordered set $\hat{k}=\left(\hat{k}_{1}, \ldots, \hat{k}_{l}\right)=\left(k_{\sigma_{\pi_{1}}}, \ldots, k_{\sigma_{\pi_{l}}}\right)$. We denote by $\alpha=\left(\lambda_{1}, \ldots, \lambda_{l}\right)$ the signs of the energies for the cut under consideration. $\lambda_{j}=1$ means that we consider the residue with positive energy with respect to the chosen orientation of the edge $e_{\sigma_{\pi_{j}}} . \hat{k}$ and $\tilde{k}$ are both bases of independent loop momenta, hence they are related by

$$
\hat{k}_{i}=\sum_{j=1}^{l} \Sigma_{i j} \tilde{k}_{j}+q_{i}
$$

with $\Sigma_{i j} \in\{-1,0,1\}$ and $q_{i}$ depending only on the external momenta. This defines the $l \times l$-signature matrix $\Sigma$. We denote by $\Sigma^{(j)}$ the $j \times j$ matrix obtained from $\Sigma$ by deleting the rows and columns $(j+1), \ldots, l$. In order to compute the residues, we may temporarily assume that the imaginary parts of all internal masses are large and strongly ordered. The final result will not depend on this assumption. After performing the contour integrations, we may remove this assumption and analytically continue to any desired (complex) kinematics. With these specifications, one obtains

$$
\frac{1}{(2 \pi i)^{l}} \int f d E_{1} \wedge \ldots \wedge d E_{l}=\sum_{\sigma \in \mathcal{C}_{\Gamma}} \sum_{\pi \in S_{l}} \sum_{\alpha \in\{1,-1\}^{l}} C_{\sigma \pi \alpha}^{\tilde{\tilde{\pi}} \tilde{\alpha} \tilde{\alpha}} \operatorname{res}\left(f, E_{\sigma}^{(\alpha)}\right)
$$

where $C_{\sigma \pi \alpha}^{\tilde{\sigma} \tilde{\pi} \tilde{\alpha}}$ is given by

$$
C_{\sigma \pi \alpha}^{\tilde{\sigma} \tilde{\pi} \tilde{\alpha}}=\prod_{i=1}^{l} \Delta^{(i)}
$$

$\Delta^{(i)}$ is zero if $\operatorname{det} \Sigma^{(i)}=0$. Otherwise, we let $\Pi^{(i)}$ be the inverse matrix of $\Sigma^{(i)}$. The quantity $\Delta^{(i)}$ is then given by

Published by the American Physical Society under the terms of the Creative Commons Attribution 4.0 International license. Further distribution of this work must maintain attribution to the author(s) and the published articles title, journal citation, and DOI. Funded by $S C O A P^{3}$. 


$$
\Delta^{(i)}=\Gamma_{i} \Pi_{i i}^{(i)} \theta\left[\Gamma_{i} \operatorname{Im}\left(\sum_{j=1}^{i} \Pi_{i j}^{(i)} \lambda_{j} m_{\sigma_{\pi_{j}}}\right)\right] .
$$

The quantities $\Delta^{(i)}$ are computed with a chosen strong ordering of the imaginary parts of the internal masses. The quantity $C_{\sigma \pi \alpha}^{\tilde{\sigma} \tilde{\pi} \tilde{\alpha}}$ is independent of this choice. Equation (2) is also valid for complex external kinematics.

One may now sum over $\pi$ and average over $\tilde{\sigma}, \tilde{\alpha}, \tilde{\pi}$ in a suitable way. We do this as follows: We group the internal propagators $D_{j}$ into chains [2]. Two propagators belong to the same chain if their momenta differ only by a linear combination of the external momenta. We denote by $n^{\text {chain }}(j)$ the number of propagators in the chain of $D_{j}$. We set

$$
N^{\text {chain }}(\sigma)=\prod_{j=1}^{l} n^{\text {chain }}\left(\sigma_{j}\right)
$$

To each graph $\Gamma$, we associate a new graph $\Gamma^{\text {chain }}$ called the chain graph by deleting all external lines and by choosing one propagator for each chain as a representative. We denote by $\left|\mathcal{C}_{\Gamma \text { chain }}\right|$ the number of spanning trees of the chain graph. We then perform a weighted average, where each term is weighted by $1 / N^{\text {chain }}(\sigma)$. We obtain

$$
\frac{1}{(2 \pi)^{l}} \int f d E_{1} \wedge \ldots \wedge d E_{l}=(-i)^{l} \sum_{\sigma \in \mathcal{C}_{\Gamma}} \sum_{\alpha=1}^{2^{l}} S_{\sigma \alpha}(-1)^{n_{\sigma}^{(\alpha)}} \operatorname{res}\left(f, E_{\sigma}^{(\alpha)}\right),
$$

with

$$
S_{\sigma \alpha}=\frac{(-1)^{l+n_{\sigma}^{(\alpha)}}}{2^{l} l !\left|\mathcal{C}_{\Gamma^{\text {chain }}}\right|} \sum_{\pi \in S_{l}} \sum_{\tilde{\sigma} \in \mathcal{C}_{\Gamma}} \sum_{\tilde{\pi} \in S_{l}} \sum_{\tilde{\alpha} \in\{1,-1\}^{l}} \frac{C_{\sigma \pi \alpha}^{\tilde{\sigma} \tilde{\pi} \tilde{\alpha}}}{N^{\text {chain }}(\sigma)} .
$$

Thus, in Eqs. (21) and (26) of this Letter, one should replace the factor $1 / 2^{l}$ with $S_{\sigma \alpha}$ under the integral and sum signs.

The factor $S_{\sigma \alpha}$ equals $1 / 2$ for all one-loop graphs, and it equals $1 / 4$ for all two-loop graphs whose underlying chain graph is a product of two one-loop tadpoles, while it equals

$$
\frac{1}{(l+1)} \frac{1}{\left(\begin{array}{c}
l \\
n_{\sigma}^{(\alpha)}
\end{array}\right)}
$$

for all two-loop graphs whose underlying chain graph is the sunrise graph, if the orientation of the cut lines is chosen the same across the cut. This agrees with Ref. [3] and covers all two-loop graphs. Equation (8) generalizes to all higher-loop graphs, whose underlying chain graph is a banana graph.

[1] Z. Capatti, V. Hirschi, D. Kermanschah, and B. Ruijl, arXiv:1906.06138.

[2] T. Kinoshita, J. Math. Phys. (N.Y.) 3, 650 (1962).

[3] S. Caron-Huot, J. High Energy Phys. 05 (2011) 080. 